\title{
Added Value of Dynamic Contrast-Enhanced Magnetic Resonance Imaging in Predicting Response to Somatostatin Analogs in Acromegaly Patients
}

\author{
Emine Sebnem DURMAZ1 , Burak KOCAK², Pinar KADIOGLU³ ${ }^{3}$ Nil COMUNOGLU4, Mustafa Onur ULU ${ }^{5}$, \\ Naci KOCER ${ }^{1}$, Civan ISLAK ${ }^{1}$, Osman KIZILKILIC ${ }^{1}$ \\ ${ }^{1}$ Istanbul University-Cerrahpasa, Cerrahpasa Medical Faculty, Department of Radiology, Istanbul, Turkey \\ ${ }^{2}$ Istanbul Training and Research Hospital, Department of Radiology, Istanbul, Turkey \\ ${ }^{3}$ Istanbul University-Cerrahpasa, Cerrahpasa Medical Faculty, Department of Endocrinology and Metabolism, Istanbul, Turkey \\ ${ }^{4}$ Istanbul University-Cerrahpasa, Cerrahpasa Medical Faculty, Department of Pathology, Istanbul, Turkey \\ ${ }^{5}$ Istanbul University-Cerrahpasa, Cerrahpasa Medical Faculty, Department of Neurosurgery, Istanbul, Turkey
}

This study has been presented as an oral presentation at the $39^{\text {th }}$ National Radiology Congress between 6 and 11 November 2018 at Antalya, Turkey.

Corresponding author: Osman KIZILKILIC osmank@istanbul.edu.tr

\section{ABSTRACT}

AIM: To investigate the added value of dynamic contrast-enhanced magnetic resonance imaging (DCE-MRI) sequences in predicting somatostatin analog (SSA) responses in patients with acromegaly.

MATERIAL and METHODS: This study included 55 active acromegaly patients with macroadenoma. Mean and maximum signal intensities were measured using region of interests in T2-weighted (T2W) and DCE-MRI sequences. Semi-quantitative values indicating relative signal intensity ratios and contrast-enhanced kinetics were obtained. Bivariate and multivariate analyses were used to determine whether the pathological granulation pattern of adenomas (dense versus others) was associated with patients' demographic variables and semi-quantitative MRI parameters.

RESULTS: Three parameters formed the logistic model, $\chi 2(3)=23.278, p<0.0001$ : age (odds ratio [OR] $=1.08$ ), hypointensity of adenomas in T2W images (OR=15.45), and high maximum enhancement ratio in the second interval $\left(E R 2_{\text {max }}\right)$ values $(O R=2195.74)$. The overall accuracy of this model was $85.45 \%$ with an area under the curve of 0.880 . Sensitivity, specificity, positive predictive, and negative predictive values of the model were $68.75 \%, 92.31 \%, 78.58 \%$, and $87.8 \%$, respectively.

CONCLUSION: In patients with newly diagnosed acromegaly, the model created based on the relative T2W signal intensity, patient's age, and $\mathrm{ER} 2_{\text {max }}$ parameter from DCE-MRI sequences might be used to more accurately predict SSA responses.

KEYWORDS: Acromegaly, Pituitary gland, Adenoma, Somatostatin, Magnetic resonance imaging

\section{INTRODUCTION}

A Ithough the first-line treatment option for acromegaly is trans-sphenoidal resection of the adenoma, complete cure rate is achieved in approximately $70 \%$ of cases even at experienced and high volume centers $(17,20)$. Conversely, the complete cure rate is $<70 \%$ when the adenoma is invasive in nature. Medical therapy is recommended in patients with a persistent disease postoperatively or in those who are at high risk for surgery (22). The standard option among medical therapies is first-generation somatostatin analogs (SSA). In the majority of patients, tumor size and growth hormone (GH) levels decrease after SSA treatment $(1,6,18,23)$. In patients
Emine Sebnem DURMAZ (1) : 0000-0002-1840-2361

Burak KOCAK

Pinar KADIOGLU
(1D) : 0000-0002-7307-396X

(D) : $0000-0002-8329-140 \mathrm{X}$
Nil COMUNOGLU (1D : 0000-0003-3573-2394

Mustafa Onur ULU (D): 0000-0002-6256-0869

Naci KOCER (1) : 0000-0003-0884-6492
Civan ISLAK

(1) : 0000-0002-3626-6244

Osman KIZILKILIC (1) : 0000-0002-6620-8934 
who are highly at risk for surgery, preoperative treatment with SSA may decrease the tumor size and eliminate or decrease tumor invasion to adjacent tissues. As a consequence, these favorable results may reduce the surgical risk. Furthermore, anesthetic complications are less likely to occur in patients preoperatively treated with SSA $(3,7,30)$. However, one-third of patients are resistant to the first-generation SSAs (21).

Factors predicting the responses to the first-generation SSA treatment are highly important in patients who are planning to undergo medical treatment due to the high economic cost of SSA treatment. Although the granulation pattern of surgically resected tumors, somatostatin receptors, and aryl hydrocarbon receptor-interacting protein expression predict the responses to SSA treatment, these parameters were derived from surgical materials $(8,14-16,31)$. Because these parameters are not available in patients newly diagnosed with acromegaly, magnetic resonance imaging (MRI) findings have a great potential to predict the responses to SSA treatment. Current literature involves strong evidence that T2-weighted (T2W)-hypointensity in GH-secreting pituitary adenomas have better responses to either primary or postoperative SSA treatment $(13,25,26,29)$. The granulation pattern of $\mathrm{GH}-$ secreting adenomas is also correlated with signal intensities on T2W images. Dense granulation pattern has been demonstrated as a strong predictor of better responses to SSA treatment (4).

To the best of our knowledge, no conventional MRI data were available in the literature, which suggest the predictors of treatment response other than low signal intensity on T2W images. Therefore, this study aimed to investigate the added value of dynamic contrast-enhanced magnetic resonance imaging (DCE-MRI) in predicting the response to SSA treatment in patients with acromegaly.

\section{MATERIAL and METHODS}

\section{Patients}

This study was approved by the local ethical committee that waived the requirement of obtaining an informed consent.

This study included 55 patients with newly diagnosed acromegaly according to clinical findings and biochemical analyses from 2009 to 2017 in our institution. Exclusion criteria were as follows: (i) the absence of pituitary DCEMRI performed in our center at the time of diagnosis; (ii) radiologically diagnosed microadenomas (maximum diameter of $<10 \mathrm{~mm}$ ); (iii) radiological or clinical diagnosis of pituitary apoplexy or bleeding; (iv) radiologically purely cystic adenomas; (v) absence or focal immunohistochemical staining in pathologic examination; and (vi) patients without pure $\mathrm{GH}-$ secreting adenomas.

Patient's age, GH levels, insulin-like growth factor-1 (IGF1) levels, tumor size at the time of diagnosis, preoperative medical treatment history, histopathological examinations, and biochemical remission status were obtained from the patient's archive files.
Acromegaly diagnosis was biochemically made based on elevated age-adjusted IGF-1 levels and GH nadir of $>1 \mu \mathrm{g} / \mathrm{L}$ on an oral glucose tolerance test.

\section{MRI Technique}

MRI scans were performed with a 1.5 Tesla MRI unit (Siemens, Magnetom Avanto) using a 12-channel head coil.

For conventional MRI, coronal T2-weighted (T2W; repetition time [TR]: $2090 \mathrm{~ms}$, echo time [TE]: $104 \mathrm{~ms}$, slice thickness [ST]: $2.5 \mathrm{~mm}$, field of view [FOV]: $180 \times 180 \mathrm{~mm}$, number of excitation [NEX]: 4, and matrix: $224 \times 320$ ), sagittal T1-weighted (T1W; TR: 350 ms, TE:10 ms, ST: 3 mm, FOV: 190×190 mm, NEX: 2, and matrix: 224×320), coronal T1W (TR: $300 \mathrm{~ms}$, TE:10 ms, ST: 2.5 mm, FOV: 190×190 mm, NEX: 3, and matrix: $192 \times 192)$ were used.

Dynamic contrast-enhanced MRI was performed after the administration of gadopentetate dimeglumine $(0.1 \mathrm{mmol} /$ $\mathrm{kg}$ ) intravenously. After administering the contrast medium, coronal and sagittal T1W images were taken (TR: $448 \mathrm{~ms}$, TE:10ms, ST: 3 mm, FOV: 190×190 mm, NEX: 2, and matrix: $224 \times 320)$. Post-contrast T1W images were obtained in three phases at 40 -s intervals.

\section{Image Analysis}

Preoperative and pretreatment MRI scans were assessed by one radiologist and a four-year radiology resident. The mean and maximum signal intensities were measured using manually drawn region of interests (ROI) in two consecutive slices in T2W, pre-contrast T1W, and all three phases of DCEMRI. The ROIs were placed onto (i) the largest solid portion of macroadenomas in all sequences; (ii) in the normal white and gray matters of the temporal lobe in a coronal T2W sequence; and (iii) in the normal white matter of the temporal lobe in pre and post-contrast T1W sequences (Figure 1A-E). The normal pituitary gland was not used for measurements because it is not visible in all patients due to large tumors. The semiquantitative values of DCE-MRI were calculated from formulas defined in Table I and Figure 2. Based on the measurements, relative $\mathrm{T} 2 \mathrm{~W}$ signal intensity $(\mathrm{rSI})$ in the adenomas were grouped as follows: (i) hypointense $(n=12)$, if the adenoma is equal or less than the mean signal intensity in the white matter; (ii) hyperintense ( $n=29$ ), if it is equal or higher than the mean signal intensity in the gray matter; and (iii) isointense $(n=14)$, if the mean signal intensity value is between the white and gray matters (13).

\section{Biochemical Response}

The SSA treatment was initiated in patients with $\mathrm{GH}$ levels of $>1 \mu \mathrm{g} / \mathrm{l}$ or elevated age-adjusted IGF-1 levels after the third month of surgery. Patients were considered resistant to SSA treatment when $\mathrm{GH}$ levels are $>1 \mu \mathrm{g} / \mathrm{l}$ or elevated age-adjusted IGF levels after 6 months of octreotide (40 mg per 28 days) or lanreotide (120 mg per 28 days) therapy.

\section{Immunohistochemistry}

Our reference standard was the official institutional immunohistochemistry report. According to the staining characteristics using monoclonal cytokeratin antibody, the adenomas were 

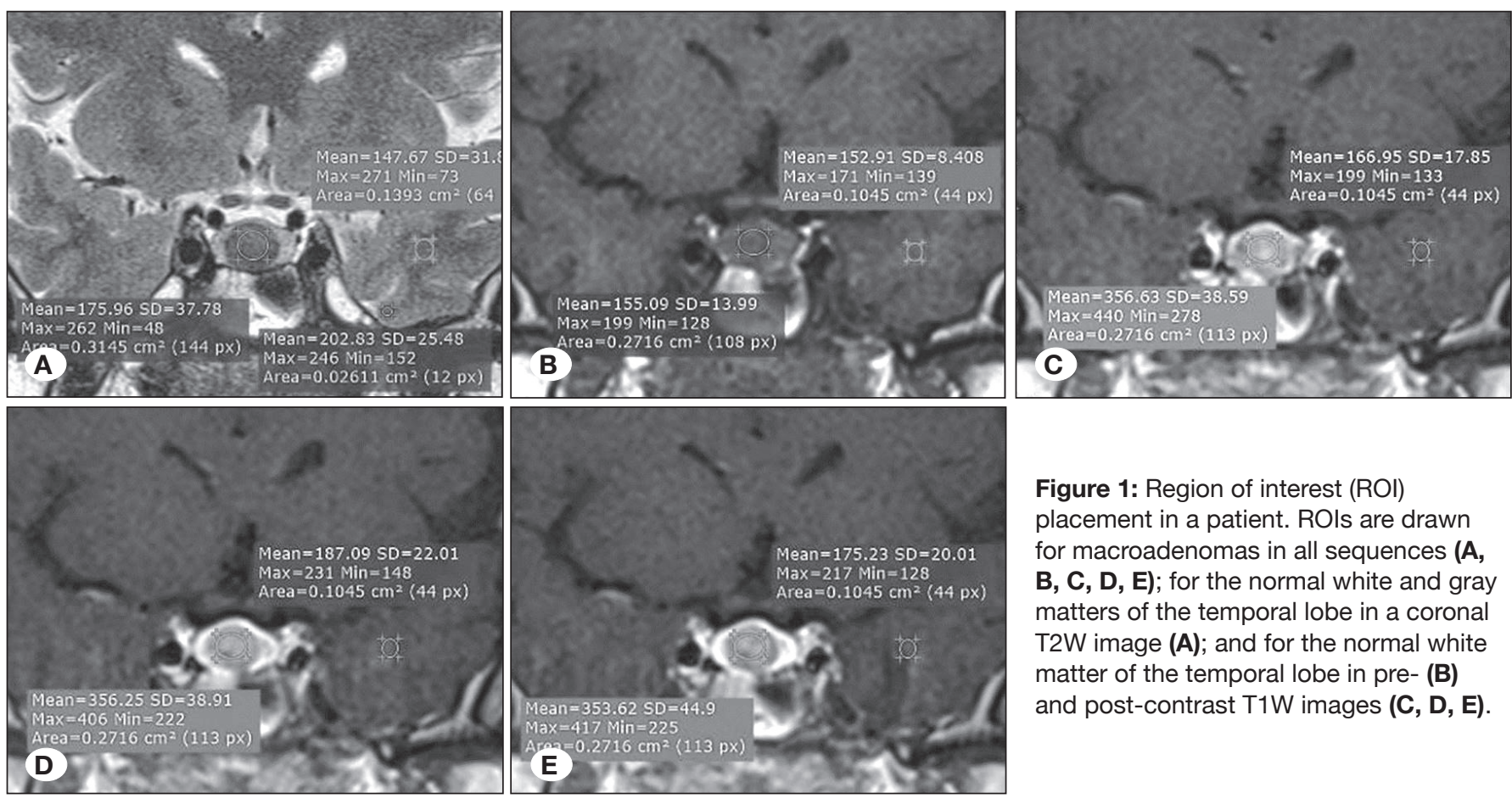

Figure 1: Region of interest (ROI) placement in a patient. ROls are drawn for macroadenomas in all sequences $(A$, B, C, D, E); for the normal white and gray matters of the temporal lobe in a coronal T2W image (A); and for the normal white matter of the temporal lobe in pre- (B) and post-contrast T1W images (C, D, E).

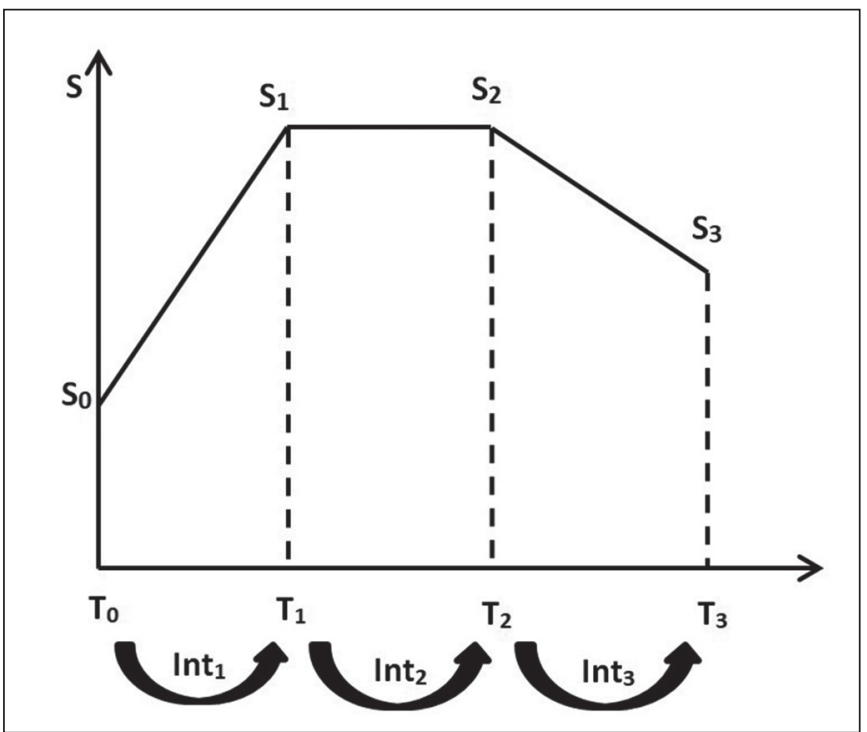

Figure 2: Simplified drawing showing a representative contrastenhanced kinetics in pituitary DCE-MRI. S, signal intensity; $\mathbf{S}_{0}$, precontrast signal intensity; $\mathbf{S}_{1-2-3}$, signal intensity at the consecutive contrast-enhanced phases; $\mathbf{T}_{0-1-2-3}$, timing of the phases; Int $\mathbf{1 - 2 - 3}$; time intervals between post-contrast phases.

divided into three groups as densely, transitional and sparsely granulated (24). For the following statistical classifications, the adenomas were then grouped as densely granulated and others (transitional and sparse).

\section{Statistical Analysis}

MedCalc Statistical Software version 18 (MedCalc Software bvba, Ostend, Belgium) and SPSS version 20 (SPSS inc.,
Chicago, IL, USA) were used for data analysis. A Chi-square test along with Phi $(\phi)$ coefficient (mean square contingency coefficient) was performed in 24 patients who were not in biochemical remission at 3 months postoperatively to validate whether our patient population is consistent with the literature regarding the positive relationship between the responses to SSA treatment (or remission) and pathological dense granulation pattern. The analysis revealed the substantial relationship, $\mathrm{p}<0.05$.

Intra-class correlation (ICC) coefficient was used to determine interobserver variability of $\mathrm{ROI}$ measurements. Only variables with ICC of $>0.81$ that represents very good to excellent agreement were included for further analyses. Consensus data were used for further analyses, which was the average of two consecutive measurements by both observers.

The Kruskal-Wallis test was used to analyze group differences between continuous variables based on the granulation pattern. The Chi-square test was used to identify group differences in proportions between categorical variables based on the granulation pattern.

To determine whether preoperative SSA treatment is associated with granulation pattern or not, a Chi-square test was used to test the homogeneity of granulation pattern between patients who underwent preoperative SSA treatment and those who directly underwent surgery. This was also further supported by the rank-biserial correlation analysis.

To create a predictive model for responses to preoperative SSA treatment using the granulation pattern (dense versus others), the multivariate analysis was conducted with logistic regression. All variables with $\mathrm{p}<0.10$ in the bivariate analyses were included in the logistic regression analysis to ascertain 
Table I: T2W MRI and DCE-MRI Parameters

\begin{tabular}{ll}
\hline Parameter from T2W MRI & \\
\hline rSIR T2 & T2S $_{\text {adenoma }} / \mathrm{T}^{2} \mathrm{~S}_{\text {twm-tgm }}$ \\
\hline Parameters from DCE-MRI & \\
\hline
\end{tabular}

First interval parameters:

\begin{tabular}{lc}
\hline ER1 & $\mathrm{S}_{1}-\mathrm{S}_{0} / \mathrm{S}_{0}$ \\
\hline Slope1 & $\mathrm{S}_{1}-\mathrm{S}_{0} / \mathrm{T}_{1}-\mathrm{T}_{0}$ \\
\hline
\end{tabular}

Second interval parameters:

\begin{tabular}{lc}
\hline ER2 & $\mathrm{S}_{2}-\mathrm{S}_{1} / \mathrm{S}_{1}$ \\
\hline Slope2 & $\mathrm{S}_{2}-\mathrm{S}_{1} / \mathrm{T}_{2}-\mathrm{T}_{1}$ \\
\hline Third interval parameters: & \\
\hline ER3 & $\mathrm{S}_{3}-\mathrm{S}_{2} / \mathrm{S}_{2}$ \\
\hline Slope3 & $\mathrm{S}_{3}-\mathrm{S}_{2} / \mathrm{T}_{3}-\mathrm{T}_{2}$ \\
\hline
\end{tabular}

Peak parameters:

\begin{tabular}{lc}
$T_{\text {peak }}$ & $\begin{array}{c}\text { signal intensity achieves } \\
\text { its maximum value first }\end{array}$ \\
\hline$r S I R_{\text {peak }}$ & $S_{\text {peak }} S_{\text {twm }}$ \\
\hline$E R_{\text {peak }}$ & $S_{\text {peak }}-S_{0} / S_{0}$ \\
\hline Slope $_{\text {peak }}$ & $S_{\text {peak }}-S_{0} / T_{\text {peak }}$ \\
\hline
\end{tabular}

Wash-out parameters

\begin{tabular}{lc} 
ER $_{\text {wash }}$ & $\mathrm{S}_{\text {peak }}-\mathrm{S}_{3} / \mathrm{S}_{\text {peak }}$ \\
\hline Slope $_{\text {wash }}$ & $\mathrm{S}_{\text {peak }}-\mathrm{S}_{3} / \mathrm{T}_{3}-\mathrm{T}_{\text {peak }}$ \\
\hline
\end{tabular}

Each parameter in the table was calculated using both mean and maximum signal intensity ratios. rSIR, relative signal intensity ratio; ER, enhancement ratio; $\mathbf{T} 2 \mathrm{~S}$, signal intensity in T2 weighted images; TWM, white matter of temporal lobe; TGM, temporal grey matter; $\boldsymbol{S}_{o}$, initial signal intensity of the adenoma before contrast medium administration; $\boldsymbol{S}_{1}$, signal intensity of the adenoma at the first postcontrast scan; $S_{2}$, signal intensity of the adenoma at the second post-contrast scan; $\mathbf{S}_{3}$, signal intensity of the adenoma at the third post-contrast scan; $\boldsymbol{T}_{1}$, time from the start of the contrast medium administration to the completion of the first scan (40sec); $\boldsymbol{T}_{2}:$ time from the start of the contrast medium administration to the completion of the second scan $(80 \mathrm{sec}) ; \boldsymbol{T}_{3}$, time from the start of the contrast medium administration to the completion of the third scan (120sec).

their possible effects on the likelihood that participants respond to the preoperative SSA treatment. An automatic stepwise selection method in combination with forward selection and backward elimination was used to create the final model.

Receiver operating characteristic (ROC) curve was plotted to determine cut-off values for the predictive variables (for each one and then combined) obtained in the logistic regression model. Youden indices were calculated and used to determine the optimal cut-off values of diagnostic accuracy parameters. Furthermore, pairwise comparisons of ROC curves were also performed in order to further assess and compare the possible predictive contributions of parameters and final model.

A $p<0.05$ indicated statistical significance for all of the abovementioned tests in all data analysis levels.

\section{RESULTS}

A total of 55 patients were included in the study, with the mean age of 39.8 (min-max, 19-65) years, female-to-male ratio of $36 / 19$, and mean tumor volume of $3287 \mathrm{~mm}^{3}$ (median, 1389.1 $\mathrm{mm}^{3} ; 2^{\text {th }}-75^{\text {th }}$ percentile, $\left.738.6-4673.7 \mathrm{~mm}^{3}\right)$. The mean $\mathrm{GH}$ and IGF-1 levels and IGF-1 ratio (IGF-1/age-adjusted upper limit of normal IGF-1 level) were $24.2 \mu \mathrm{g} / \mathrm{l}$ (median, $24.2 \mu \mathrm{g} / \mathrm{l}$; $25^{\text {th }}-75^{\text {th }}$ percentile, 6.7-33.8 $\mu \mathrm{g} / \mathrm{l}$ ), $814.8 \mathrm{mmol} / \mathrm{l}$ (median, $814.8 \mathrm{mmol} / \mathrm{l} ; 25^{\text {th }}-75^{\text {th }}$ percentile, 671.3-945 $\left.\mathrm{mmol} / \mathrm{l}\right)$, and 3 (median, 2.7; 25 $5^{\text {th }}-75^{\text {th }}$ percentile, 2.3-3.4), respectively. Among the 55 adenomas, 30 (54.5\%) were classified as sparsely, 9 (16.4\%) as transitional, and 16 (29.1\%) as densely depending on the granulation pattern.

The ICC coefficients for the mean and maximum signal intensities measured in non-enhanced T1W and DCE-MRI images ranged between 0.91 and 1 . In T2W images, ICC coefficients for maximum signal intensities measured from white and gray matters ranged between 0.81 and 0.90 . Conversely, other measurements on T2W images were within the range of 0.91 to 1 . Because both readers had very good to excellent agreement (ICC coefficient of $>0.81$ ), all of the signal intensity measurements were included in calculating the parameters presented in Table I.

As assessed using the Chi-square test, the granulation pattern distribution between the patients preoperatively treated with SSA $(n=19)$ and those directly operated was not significantly different, $\chi 2(2)=0.726, \quad(p=0.696)$. This was also supported by the rank-biserial correlation analysis, $r_{r b}(53)=-0.0174$, $(p=0.899)$.

No statistically significant difference was found in gender distribution $\chi 2(2)=1.813,(p=0.404)$, tumor volume $(p=0.365)$, IGF-1 level $(p=0.391)$, and IGF-1 ratio $(p=0.05)$ between the three granulation groups. A statistically significant difference was found in age $(p=0.036)$ and $\mathrm{GH}$ level $(p=0.040)$ between the groups. In pairwise comparisons, no statistically significant difference was noted in age between the pairs ( $p>0.05)$. Conversely, GH level differences between sparse and transitional groups were statistically significant $(p=0.036)$. Regarding the parameters calculated from the T2W data, a statistically significant difference was observed in $\mathrm{T} 2 \mathrm{~W}$ intensity of adenomas, $\chi 2(4)=15.794, \quad(p=0.003)$. Figure 3 shows the frequency chart for T2W intensity of adenomas based on the granulation pattern for pairwise comparisons. With regard to the dynamic contrast-enhanced T1W image data, the parameter of enhancement ratio in the second interval (ER2 $\left.{ }_{\text {max }}\right)$ was statistically significantly different between the groups $(p=0.035)$. In pairwise comparisons, differences in $\mathrm{ER} 2_{\text {max }}$ between transitional and dense groups were also statistically significant $(p=0.038)$. 
Table II: Results from Logistic Regression Analysis

\begin{tabular}{lcccccc}
\hline Variables & Coefficient & Std. Error & Wald & Odds ratio & 95\% Cl & p \\
\hline Age & 0.075877 & 0.038438 & 3.8967 & 1.0788 & 1.0005 to 1.1632 & $\mathbf{0 . 0 4 8 4}$ \\
\hline T2 hypointensity & 2.73775 & 0.87754 & 9.7333 & 15.4522 & 2.7670 to 86.2910 & $\mathbf{0 . 0 0 1 8}$ \\
\hline ER2 maximum & 7.69428 & 3.40359 & 5.1105 & 2195.7423 & 2.7822 to 1732895.4206 & $\mathbf{0 . 0 2 3 8}$ \\
\hline Constant & -5.47751 & 1.85802 & 8.6909 & - & - & $\mathbf{0 . 0 0 3 2}$ \\
\hline
\end{tabular}

The following ten variables were included in the multivariate analysis: age $(p=0.036), \mathrm{GH}$ level $(p=0.040), \mathrm{IGF}-1$ ratio $(p=0.05)$, T2W intensity of the adenoma $(p=0.003)$, rSIRT2 ${ }_{\text {mean }}(p=0.054), \quad r S I R-T 2_{\max }(p=0.056), E R 2_{\max } \quad(p=0.035)$, Slope2 $2_{\text {max }}(p=0.078), E R 3_{\text {mean }}(p=0.069)$, and Slope $_{\text {mean }}$ $(p=0.059)$. The final logistic regression model was statistically significant, $\chi 2(3)=23.278, p<0.0001$. The model explained $49.2 \%$ (Nagelkerke $R 2$ ) of the variance in granulation pattern and correctly classified $85.45 \%$ of cases. The model's sensitivity, specificity, positive predictive value, and negative predictive value were $68.75 \%, 92.31 \%, 78.58 \%$, and $87.8 \%$, respectively. Among the ten predictor variables, only three were statistically significant: age, T2W-hypointensity of the adenoma, and $\mathrm{ER} 2_{\text {max }}$. Increasing age (odds ratio $[\mathrm{OR}]=1.08$; $95 \%$ confidence interval $[\mathrm{Cl}]=1.0005-1.1632)$, having $\mathrm{T} 2 \mathrm{~W}$ hypointensity in the adenomas $(\mathrm{OR}=15.45 ; 95 \% \mathrm{Cl}=2.76-$ 86.29), and higher $\mathrm{ER} 2_{\max }(\mathrm{OR}=2195.74 ; 95 \% \mathrm{Cl}=2.78-$ 1732895.42) were associated with an increased likelihood of dense granulation that indicates better response to the SSA treatment. Table II presents the results of the logistic regression analysis. Table III shows the cut-off values determined using Youden indices and its relevant diagnostic parameters in detail. Figure 4 presents the ROC curves of age, $\mathrm{ER} 2_{\max }$, T2W-hypointensity of the adenomas, and the final logistic regression model. The area under the curve (AUC) of the model was $0.880(p<0.0001)$. Pairwise comparison of $R O C$ curves revealed statistically significant differences between (i) age and logistic regression model (difference between $\mathrm{AUC}=0.159$ [95\% $\mathrm{Cl}=0.027-0.0292], \mathrm{p}=0.018$ ) and (ii) T2W-hypointensity and logistic regression model (difference between $A \cup C=0.167 \quad[95 \% \mathrm{Cl}=0.005-0.330], p=0.431)$. No statistically significant difference was observed between the $\mathrm{ER} 2_{\text {max }}$ and logistic regression model (difference between $A \cup C=0.184[95 \% \mathrm{Cl}=0.001-0.370], \mathrm{p}=0.051)$, indicating that $\mathrm{ER} 2_{\text {max }}$ alone can potentially predict the response to SSA treatment with a performance as high as the model does.

\section{DISCUSSION}

The main finding in this study is a semi-quantitative evaluation of DCE-MRI as the additive value to T2W signal intensity evaluation in predicting the response to SSA treatment in patients with acromegaly. The model created using the T2W hypointensity of the adenoma, $\mathrm{ER} 2_{\max }$ parameter, and patient's age together may predict the granulation pattern of $\mathrm{GH}$-secreting adenomas with rather high overall accuracy.

Current literature indicates that in acromegaly patients with macroadenoma $(>10 \mathrm{~mm})$, preoperative SSA treatment

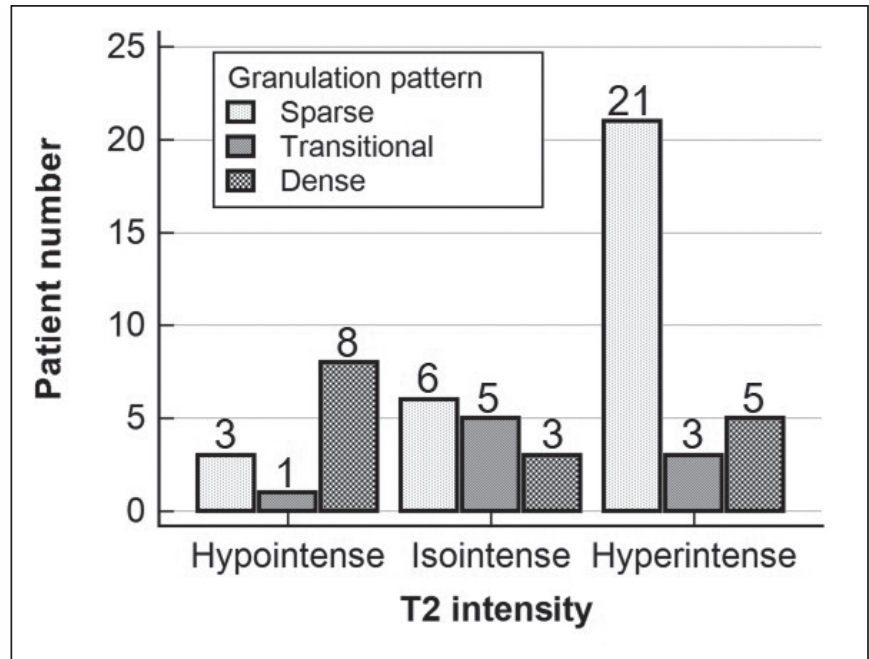

Figure 3: Frequency chart for T2W intensity of the adenoma based on the granulation pattern.

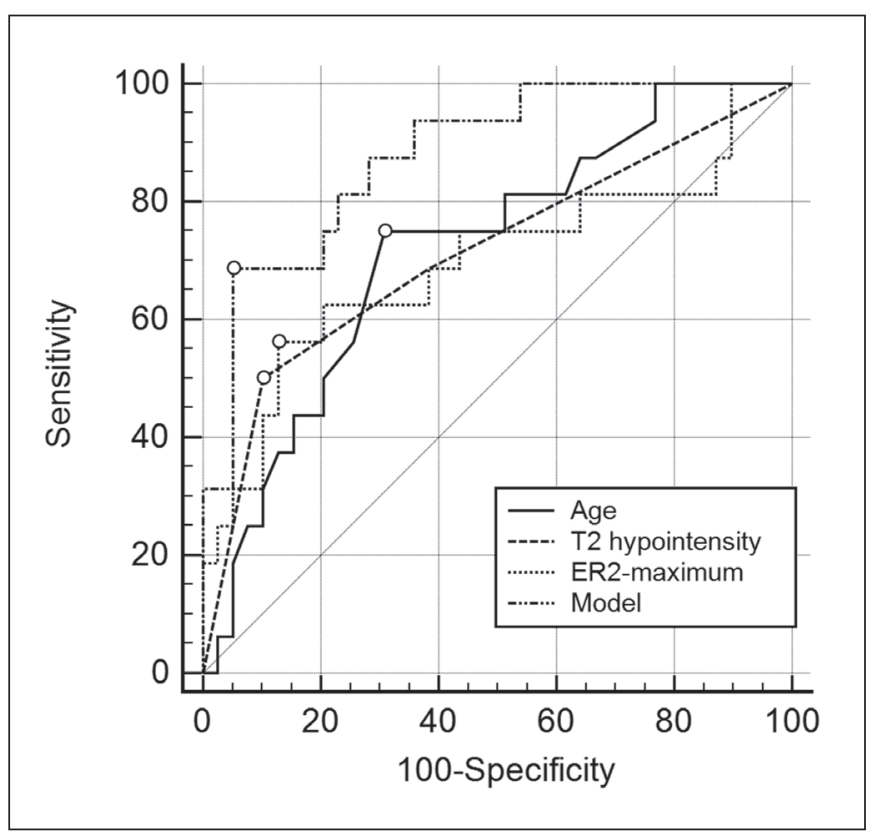

Figure 4: Receiver operating characteristic (ROC) curves of age, ER2 ${ }_{\text {max }}, T 2 W$-hypointensity of the adenomas, and the final logistic regression model. 
improves the surgical outcomes $(2,5,19,28)$. Response to SSA treatment is highly variable among patients with acromegaly. Some patients respond quite well to SSA treatment with a full biochemical remission and reduction of tumor volume $(1,6,18,23)$. In contrast, some patients are resistant to SSA treatment, which may delay the surgery and deteriorate surgical outcomes. For this reason, creating and developing the preoperative predictive models for the response to SSA treatment is of supreme importance because they may guide the treatment strategy and improve patients' prognosis. Although all evidential data is based on histological variables obtained from surgically resected adenomas, MRI, particularly the T2W sequence, appears to be a potential tool in predicting the preoperative response to SSAs $(11,12,25,26,29)$. To the best of our knowledge, no other study evaluated $\mathrm{GH}$ secreting macroadenomas with the dynamic enhancement characteristics.

Pituitary adenoma subtypes demonstrate various vascular features. Guo et al. evaluated the DCE-MRI value to differentiate ACTH-secreting pituitary adenomas from nonfunctioning adenomas. In that study, they measured the enhancement time, enhancement peak, and pre-peak slope (PPS), which showed that the PPS of ACTH-secreting pituitary adenomas were statistically significantly lower than that of non-functioning adenomas (10). Romano et al. evaluated the differences in areas of consistency in macroadenomas. Their results proved that DCE-MRI can distinguish macroadenoma's consistency with good accuracy (27). In our study, DCE-MRI was semi-quantitatively assessed with additional kinetic features such as mean or maximum enhancement ratios for each interval. Nonetheless, one (ER2 $\left.{ }_{\max }\right)$ of these additional kinetic features was included in the final multivariate logistic model. Although it is unclear, the interval kinetic parameter $\left(E R 2_{\text {max }}\right)$ may signify subtle differences in vascular kinetics of adenomas. Continued efforts are needed to validate these features.

In our study, T2W-hypointense macroadenomas are significantly associated with a dense granulation pattern consistent with previous studies, indicating better responses to preoperative SSA treatment. Hagiwara et al. demonstrated T2W hyperintensity is shown to be more frequently found in sparsely granulated adenomas than densely granulated (11). Several studies in the literature assessed the relationship between preoperative use of SSA and T2W signal intensity of adenomas $(13,26)$.

In three studies, T2W signal intensity has been quantitatively evaluated. The lower signal intensity values in T2W images were correlated with higher decrease in GH and IGF-1 levels in patients with SSA treatment $(12,25,29)$. Although the threshold of T2W signal intensity ratio to predict response to SSA treatment was measured in two different studies, these values cannot be compared due to different tissues used as reference. One of these studies did not reveal any differences in T2W signal intensity of adenomas when evaluated quantitatively or qualitatively (12).

In this study, the granulation pattern and patient's age were statistically significant in both bivariate and multivariate 
analyses. Increasing age is found to be associated with dense granulation pattern, indicating that older patients respond better to SSA treatment than younger ones. This finding is also concordant with studies in the literature (29).

A number of important limitations should be considered. First, during the evaluation of response to SSA treatment, the granulation pattern was used as the reference, which may be a limitation instead of biochemical remission criteria. However, in successfully operated patients without residual tumor, biochemical remission is an expected outcome; therefore, it would not be possible to evaluate the response of SSA treatment. Therefore, the biochemical response status was correlated with the granulation pattern in 24 patients who were not in biochemical remission at 3 months postoperatively. Current literature also involves studies that demonstrate the relationship between better response to SSA treatment and dense granulation pattern (4). Second, the pituitary DCEMRI protocol in our center includes three post-contrast phases. Using different protocols might provide new valuable parameters. Third, all patients were included regardless of their preoperative SSA treatment history. To assess whether the granulation pattern changes after the therapy or not, the distribution of granulation pattern was assessed and found no statistically significant difference between patients treated and untreated with SSA treatment preoperatively. In the literature, as far as we know, only one study analyzed the granulation pattern distribution in a few patients with available adenoma tissue from surgeries before and after SSA treatment (9). Their findings were consistent with those of our study. Fourth, a Bonferroni correction for multivariate analysis was not performed considering the potential risk of Type II error. Finally, our hospital is a referral center for pituitary surgery, although many patients had follow-up data, those who underwent MRI in different centers, with microadenomas or pure cystic adenomas, with hemorrhagic adenomas, diagnosed with pituitary apoplexy, without pure GH-secreting adenomas, and with incomplete immunohistochemically staining adenomas were excluded. Therefore, the number of included patients is rather small.

\section{- CONCLUSION}

Our study demonstrated that the model created based on the relative T2W signal intensity, patient's age, and $\mathrm{ER} 2_{\max }$ parameters from DCE-MRI sequences might be used to more accurately predict SSA responses in patients with newly diagnosed acromegaly.

\section{REFERENCES}

1. Annamalai AK, Webb A, Kandasamy N, Elkhawad M, Moir S, Khan F, et al: A comprehensive study of clinical, biochemical, radiological, vascular, cardiac, and sleep parameters in an unselected cohort of patients with acromegaly undergoing presurgical somatostatin receptor ligand therapy. J Clin Endocrinol Metab 98:1040-1050, 2013
2. Bacigaluppi S, Gatto F, Anania P, Bragazzi NL, Rossi DC, Benvegnu G, et al: Impact of pre-treatment with somatostatin analogs on surgical management of acromegalic patients referred to a single center. Endocrine 51:524-533, 2016

3. Barkan AL, Lloyd R V., Chandler WF, Hatfield MK, Gebarski SS, Kelch RP, et al: Preoperative treatment of acromegaly with long-acting somatostatin analog sms 201-995: Shrinkage of invasive pituitary macroadenomas and improved surgical remission rate. J Clin Endocrinol Metab 67:1040-1048, 1988

4. Bhayana S, Booth GL, Asa SL, Kovacs K, Ezzat S: The implication of somatotroph adenoma phenotype to somatostatin analog responsiveness in acromegaly. J Clin Endocrinol Metab 90:6290-6295, 2005

5. Carlsen SM, Lund-Johansen M, Schreiner T, Aanderud S, Johannesen O, Svartberg J, Cooper JG, Hald JK, Fougner SL, Bollerslev J; Preoperative Octreotide Treatment of Acromegaly study group: Preoperative octreotide treatment in newly diagnosed acromegalic patients with macroadenomas increases cure short-term postoperative rates: A prospective, randomized trial. J Clin Endocrinol Metab 93(8):2984-2990, 2008

6. Carlsen SM, Svartberg J, Schreiner T, Aanderud S, Johannesen À, Skeie S, et al: Six-month preoperative octreotide treatment in unselected, de novo patients with acromegaly: Effect on biochemistry, tumour volume, and postoperative cure. Clin Endocrinol (Oxf) 74:736-743, 2011

7. Colao A, Ferone D, Cappabianca P, Del Basso De Caro ML, Marzullo P, Monticelli A, et al: Effect of octreotide pretreatment on surgical outcome in acromegaly. J Clin Endocrinol Metab 82:3308-3314, 1997

8. Ferone D, De Herder WW, Pivonello R, Kros JM, Van Koetsveld PM, De Jong T, et al: Correlation of in vitro and in vivo somatotropic adenoma responsiveness to somatostatin analogs and dopamine agonists with immunohistochemical evaluation of somatostatin and dopamine receptors and electron microscopy. J Clin Endocrinol Metab 93:1412-1417, 2008

9. Fougner SL, Casar-Borota O, Heck A, Berg JP, Bollerslev J: Adenoma granulation pattern correlates with clinical variables and effect of somatostatin analogue treatment in a large series of patients with acromegaly. Clin Endocrinol (Oxf) 76:96-102, 2012

10. Guo Q, Young WF, Erickson D, Erickson B: Usefulness of dynamic MRI enhancement measures for the diagnosis of ACTH-producing pituitary adenomas. Clin Endocrinol (Oxf) 82:267-273, 2015

11. Hagiwara A, Inoue $Y$, Wakasa K, Haba T, Tashiro T, Miyamoto $\mathrm{T}$ : Comparison of growth hormone-producing and nongrowth hormone-producing pituitary adenomas: Imaging characteristics and pathologic correlation. Radiology 228:533-538, 2003

12. Heck A, Emblem KE, Casar-Borota O, Bollerslev J, Ringstad G: Quantitative analyses of T2-weighted MRI as a potential marker for response to somatostatin analogs in newly diagnosed acromegaly. Endocrine 52:333-343, 2016 
13. Heck A, Ringstad G, Fougner SL, Casar-Borota O, Nome T, Ramm-Pettersen J, et al: Intensity of pituitary adenoma on T2weighted magnetic resonance imaging predicts the response to octreotide treatment in newly diagnosed acromegaly. Clin Endocrinol (Oxf) 77:72-78, 2012

14. lacovazzo D, Carlsen E, Lugli F, Chiloiro S, Piacentini S, Bianchi A, et al: Factors predicting pasireotide responsiveness in somatotroph pituitary adenomas resistant to first-generation somatostatin analogues: An immunohistochemical study. Eur J Endocrinol 174:241-250, 2016

15. Jaffrain-Rea ML, Angelini $M$, Gargano $D$, Tichomirowa MA, Daly AF, Vanbellinghen JF, et al: Expression of aryl hydrocarbon receptor $(\mathrm{AHR})$ and $\mathrm{AHR}$-interacting protein in pituitary adenomas: Pathological and clinical implications. Endocr Relat Cancer 16:1029-1043, 2009

16. Kasuki L, Neto LV, Wildemberg LEA, Colli LM, De Castro M, Takiya CM, et al: AIP expression in sporadic somatotropinomas is a predictor of the response to octreotide LAR therapy independent of SSTR2 expression. Endocr Relat Cancer 19:L25-L29, 2012

17. Katznelson L, Laws ER, Melmed S, Molitch ME, Murad MH, Utz A, et al: Acromegaly: An endocrine society clinical practice guideline. J Clin Endocrinol Metab 99:3933-3951, 2014

18. Luque-Ramírez M, Portoles GR, Varela C, Albero R, Halperin I, Moreiro J, et al: The efficacy of octreotide lar as firstline therapy for patients with newly diagnosed acromegaly is independent of tumor extension: Predictive factors of tumor and biochemical response. Horm Metab Res 42:38-44, 2010

19. Mao ZG, Zhu YH, Tang HL, Wang DY, Zhou J, He DS, et al: Preoperative lanreotide treatment in acromegalic patients with macroadenomas increases short-term postoperative cure rates: A prospective, randomised trial. Eur J Endocrinol 162:661-666, 2010

20. Melmed S: Acromegaly pathogenesis and treatment. J Clin Invest 119:3189-3202, 2009

21. Melmed S, Colao A, Barkan A, Molitch M, Grossman AB, Kleinberg D, et al: Guidelines for acromegaly management: An update. J Clin Endocrinol Metab 94:1509-1517, 2009

22. Melmed S, Jackson I, Kleinberg D, Klibanski A: Current treatment guidelines for acromegaly. J Clin Endocrinol Metab 83:2646-2652, 1998
23. Mercado M, Borges F, Bouterfa H, Chang TC, Chervin A, Farrall AJ, et al: A prospective, multicentre study to investigate the efficacy, safety and tolerability of octreotide LAR $®$ (longacting repeatable octreotide) in the primary therapy of patients with acromegaly. Clin Endocrinol (Oxf) 66:859-868, 2007

24. Obari A, Sano T, Ohyama K, Kudo E, Qian ZR, Yoneda A, et al: Clinicopathological features of growth hormone-producing pituitary adenomas: Difference among various types defined by cytokeratin distribution pattern including a transitional form. Endocr Pathol 19:82-91, 2008

25. Potorac I, Petrossians P, Daly AF, Alexopoulou O, Borot S, Sahnoun-Fathallah M, et al: T2-weighted MRI signal predicts hormone and tumor responses to somatostatin analogs in acromegaly. Endocr Relat Cancer 23:871-881, 2016

26. Puig-Domingo M, Resmini E, Gomez-Anson B, Nicolau J, Mora M, Palomera E, et al: Magnetic resonance imaging as a predictor of response to somatostatin analogs in acromegaly after surgical failure. J Clin Endocrinol Metab 95:4973-4978, 2010

27. Romano A, Coppola V, Lombardi M, Lavorato L, Di Stefano D, Caroli E, et al: Predictive role of dynamic contrast enhanced T1-weighted MR sequences in pre-surgical evaluation of macroadenomas consistency. Pituitary 20:201-209, 2017

28. Shen M, Shou X, Wang Y, Zhang Z, Wu J, Mao Y, et al: Effect of presurgical long-acting octreotide treatment in acromegaly patients with invasive pituitary macroadenomas: A prospective randomized study. Endocr J 57:1035-1044, 2010

29. Shen M, Zhang Q, Liu W, Wang M, Zhu J, Ma Z, et al: Predictive value of $\mathrm{T} 2$ relative signal intensity for response to somatostatin analogs in newly diagnosed acromegaly. Neuroradiology 58:1057-1065, 2016

30. Stevenaert A, Beckers A: Presurgical octreotide treatment in acromegaly. Acta Endocrinol (Copenh) 129 Suppl 1:18-20, 1993

31. Taboada GF, Luque RM, Neto LV, Machado E de O, Sbaffi BC, Domingues RC, et al: Quantitative analysis of somatostatin receptor subtypes (1-5) gene expression levels in somatotropinomas and correlation to in vivo hormonal and tumor volume responses to treatment with octreotide LAR. Eur J Endocrinol 158:295-303, 2008 